\title{
Measurements of the Spectral Energy Distribution of the Cosmic Infrared Background
}

\author{
Matthieu Bethermin* \\ Institut d'Astrophysique Spatiale, Univ. Paris Sud 11 \& CNRS, Orsay \\ E-mail: matthieu.bethermineias.u-psud.fr
}

\section{Hervé Dole}

Institut d'Astrophysique Spatiale, Univ. Paris Sud $11 \&$ CNRS, Orsay, and Institut Universitaire de France

E-mail: herve.doledias.u-psud.fr

The extragalactic background light (EBL) is the relic emission of all processes of structure formation in the Universe. About half of this background, called the Cosmic Infrared Background (CIB) is emitted in the $8-1000$ microns range, and peaks around 150 microns. It is due to the dust reemission from star formation processes and AGN emission. The CIB spectral energy distribution (SED) constraints the models of star formation in the Universe. It is also useful to compute the opacity of the Universe to the $\mathrm{TeV}$ photons. We present the different types of measurements of the CIB and discuss their strengths and weaknesses.

1. The absolute SED was measured by COBE, and by other experiments. These measurements are limited by the accuracy of the component separation, i.e. the foreground subtraction.

2. Robust lower limits are determined from the extragalactic number counts of infrared galaxies. These lower limits are very stringent up to 100 microns. At larger wavelengths, the rather low angular resolution of the instruments limits strongly the depth of the number counts. The "stacking" method determines the flux emitted at a given wavelength by a population detected at another wavelength, and provides stringent lower limits in the sub-mm range. It is complementary with other methods based on the statistical analysis of the map properties like the $\mathrm{P}(\mathrm{D})$ analysis.

3. Finally, upper limits can be derived from the high energy spectra of extragalactic sources. These upper limits give currently good constraints in the near- and mid-IR.

Progress have been amazing since the CIB discovery about 15 years ago: the SED is much better known, and most of it can be accounted for by galaxies (directly or indirectly). Prospects are also exciting, with fluctuation analysis with Planck \& Herschel, and forthcoming missions.

Cosmic Radiation Fields: Sources in the early Universe - CRF2010,

November 9-12, 2010

Desy Germany

\footnotetext{
*Speaker.
} 


\section{Introduction}

The cosmic infrared background (CIB) was detected for the first time in the late nineties in the FIRAS and DIRBE data [1 -3]. This background, lying in the mid- far-infrared and submillimeter range (usually defined between 8 and $1000 \mu \mathrm{m}$ ), represents about half [4] of the extragalactic background light (EBL), which is the relic of all the structure formation processes. The CIB emission is mainly due to the outputs of the infrared galaxies, and in a minor way to the obscured active galactic nuclei (AGN) [5, 6]. It peaks near $150 \mu \mathrm{m}$.

The output of the infrared galaxies is a good probe of the star formation [7]. During a starburst, the massive stars have short lives but strong UV emissions, which are absorbed and reprocessed by the dusty environment where they formed. Infrared outputs are thus a probe of the presence of massive young stars and thus of the recent star formation in a galaxy. Dust heated by AGN also contribute to the CIB, but almost an order of magnitude fainter than starbursts [8]. The spectral energy distribution (SED) of the CIB thus constrains the star formation history and the galaxy evolution models [9-13].

\section{Direct measurements}

Direct measurements of the CIB can be performed using absolute photometry, but these measurements are affected by the accuracy of the foreground modeling. These foregrounds are the zodiacal emission [14] and the galactic cirrus [15]. The zodiacal emission is the thermal radiation of the interplanetary dust. The galactic cirrus are diffuse clouds of dust in our galaxy heated by the UV emissions of the stars. At $20 \mu \mathrm{m}$, the zodiacal light is three order of magnitude brighter than the CIB. The accuracy of the zodiacal subtraction is thus the main limitation of the absolute measurements. The galactic cirrus output have the same order of magnitude than the CIB. They can be removed accurately using the current $\mathrm{HI}$ data [16]. At larger wavelength, the cosmic microwave background have to be subtracted. The spectrum of the CMB being well known, its subtraction can be done accurately [17].

First measurements of the CIB was performed with DIRBE [3] and FIRAS [2, 15] onboard COBE. The DIRBE measurements have been updated in [4] (see discussion about DIRBE/FIRAS cross-calibration and subtraction of the zodiacal light). More recent measurements have been performed with, ISO [18], Akari [19] and Spitzer [16]. These measurements are summarized in the Fig. 1 and Table 1. Notice the large scatter in the measurements. Notice also the IRAS measurements [20] based on a fluctuation analysis.

\section{Lower limits from the number counts}

In the mid-infrared, the depth and the angular resolution of the recent infrared surveys is sufficient to resolve the main part of the CIB into sources. Stringent lower limits can thus be derived from the integration of the source counts. These lower limits were derived at $8 \mu \mathrm{m}$ using the Spitzer/IRAC data [21], at $15 \mu \mathrm{m}$ using ISO [31], Spitzer [22] and Akari [32] data, and at $24 \mu \mathrm{m}$ [23]. Estimations of the total contribution of the galaxies to the CIB was done by extrapolating the 


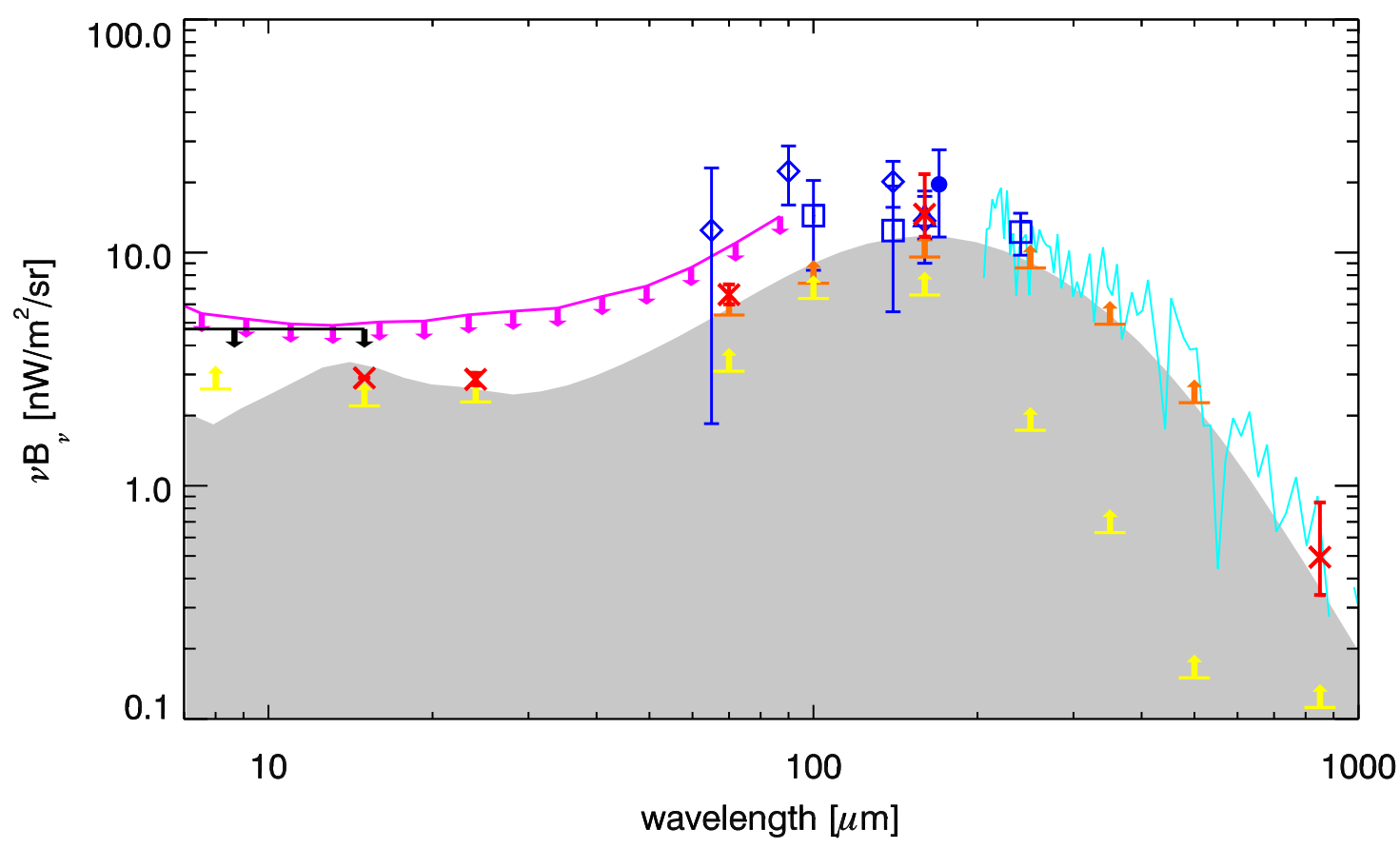

Figure 1: Spectral energy distribution of the cosmic infrared background. Yellow arrows: Lower limits from resolved source counts at $8 \mu \mathrm{m}$ [21], $15 \mu \mathrm{m}$ [22], $24 \mu \mathrm{m}, 70 \mu \mathrm{m}$ [23], $100 \mu \mathrm{m}, 160 \mu \mathrm{m}$ [24], 250 $\mu \mathrm{m}$, $350 \mu \mathrm{m}$ and $500 \mu \mathrm{m}$ [25] and $850 \mu \mathrm{m}$ [26]. Orange arrows: Lower limits from stacking analysis at $70 \mu \mathrm{m}$ [23], $100 \mu \mathrm{m}, 160 \mu \mathrm{m}$ [24], $250 \mu \mathrm{m}, 350 \mu \mathrm{m}$ and $500 \mu \mathrm{m}$ [27]. Red crosses: Extrapolated total contribution of the galaxies to the CIB at $15 \mu \mathrm{m}$ [22], $24 \mu \mathrm{m}, 70 \mu \mathrm{m}, 160 \mu \mathrm{m}$ [23] and $850 \mu \mathrm{m}$ [28]. Blue square: DIRBE absolute measurements [3]. Blue diamonds: Akari absolute measurements [19]. Blue triangle: MIPS absolute measurement at $160 \mu \mathrm{m}$ [16]. Blue circle: ISOPHOT absolute measurement at $170 \mu \mathrm{m}$ [18]. Cyan solid line: FIRAS Spectrum [15]. Black arrows: Upper limits from TeV opacity of the Universe [29]. Purple arrows: Upper limits from TeV opacity of the Universe [30]. Light grey area: CIB predicted by the Bethermin et al. model [12].

faint-end slope of the counts. Assuming the CIB is only due to the galaxies, the fraction of the CIB resolved directly is then estimated to be $\sim 80 \%$ [23].

At larger wavelengths, the angular resolution of the instruments decreases compared to wavelength, and it is harder to resolve a significant part of the CIB due to the confusion [33, 34]. For example, we resolve only $40 \%$ and $15 \%$ of the CIB at 70 and $160 \mu \mathrm{m}$, respectively with Spitzer [23]. Thanks to its $3.5 \mathrm{~m}$ diameter mirror, Herschel resolves $\sim 45 \%$ and $\sim 52 \%$ at 100 and $160 \mu \mathrm{m}$, respectively [24]. Nevertheless, this fraction decreases to $\sim 15 \%, \sim 10 \%$ and $\sim 6 \%$ at 250,350 and $500 \mu \mathrm{m}$, respectively [25]. At $850 \mu \mathrm{m}$, the opacity of the atmosphere is sufficiently low to allow observations from the ground. The counts at this wavelength resolve only 20-30\% of the CIB [26]. Deeper counts, resolving the bulk of the CIB, are derived in fields lensed by low-z galaxy clusters, where the error budget is dominated by the large scale structure (narrow field) [35, 28]. These measurements are summarized in the Fig. 1 and Table 1. 


\begin{tabular}{|c|c|c|c|c|}
\hline $\begin{array}{l}\text { Wavelength } \\
\mu \mathrm{m}\end{array}$ & Reference & $\begin{array}{l}\text { CIB level } \\
\mathrm{nW} \cdot \mathrm{m}^{-2} \cdot \mathrm{sr}^{-1}\end{array}$ & Instrument & Comments \\
\hline \multicolumn{5}{|c|}{ Absolute measurements } \\
\hline 65 & Matsuura et al. (2009) & $12.5 \pm 1.4 \pm 9.2$ & Akari & \\
\hline 90 & Matsuura et al. (2009) & $22.3 \pm 1.7 \pm 4.7$ & Akari & \\
\hline 100 & Lagache et al. (2000) & $14.4 \pm 6.0$ & DIRBE & Updated in Dole et al. (2006) \\
\hline 140 & Lagache et al. (2000) & $12.4 \pm 6.9$ & DIRBE & Updated in Dole et al. (2006) \\
\hline 140 & Matsuura et al. (2009) & $20.1 \pm 3.4 \pm 1.1$ & Akari & \\
\hline 160 & Matsuura et al. (2009) & $13.7 \pm 3.9 \pm 40.8$ & Akari & \\
\hline 160 & Penin et al. (2011) & $14.43 \pm 3$ & Spitzer/MIPS & \\
\hline 170 & Juvela et al. (2009) & $19.6 \pm 5.8 \pm 5.5$ & ISOPHOT & \\
\hline 240 & Lagache et al. (2000) & $12.3 \pm 2.5$ & DIRBE & Updated in Dole et al. (200 \\
\hline $200-1000$ & Lagache et al. (2000) & see figure & FIRAS & \\
\hline \multicolumn{5}{|c|}{ Lower limit from resolved sources } \\
\hline 8 & Fazio et al. (2004) & 5.4 & Spitzer/IRAC & QSO1700+EGS+Bootes \\
\hline 15 & Teplitz et al. (2010) & $2.2 \pm 0.2$ & Spitzer/IRS & GOODS-N and S \\
\hline 24 & Béthermin et al. (2010) & $2.29 \pm 0.09$ & Spitzer/MIPS & FIDEL+COSMOS+SWIRE \\
\hline 70 & Béthermin et al. (2010) & $3.1 \pm 0.2$ & Spitzer/MIPS & FIDEL+COSMOS+SWIRE \\
\hline 100 & Berta et al. (2010) & $6.33 \pm 1.67$ & Herschel/PACS & SDP data \\
\hline 160 & Berta et al. (2010) & $6.58 \pm 1.62$ & Herschel/PACS & SDP data \\
\hline 250 & Oliver et al. (2010) & $1.73 \pm 0.33$ & Herschel/SPIRE & SDP data \\
\hline 350 & Oliver et al. (2010) & $0.63 \pm 0.18$ & Herschel/SPIRE & SDP data \\
\hline 500 & Oliver et al. (2010) & $0.15 \pm 0.07$ & Herschel/SPIRE & SDP data \\
\hline 850 & Coppin et al. (2008) & $0.11_{-0.04}^{+0.05}$ & SCUBA & SHADES survey \\
\hline \multicolumn{5}{|c|}{ Lower limits from stacking analysis } \\
\hline 70 & Béthermin et al. (2010) & $5.4 \pm 0.4$ & Spitzer/MIPS & FIDEL+COSMOS+SWIRE \\
\hline 100 & Berta et al. (2010) & $7.4 \pm 0.5$ & Herschel/PACS & SDP data \\
\hline 160 & Berta et al. (2010) & $9.6 \pm 0.7$ & Herschel/PACS & SDP data \\
\hline 250 & Marsden et al. (2009) & $8.6 \pm 0.6$ & BLAST & biased by clustering $(\sim+9 \%)$ \\
\hline 350 & Marsden et al. (2009) & $4.9 \pm 0.3$ & BLAST & biased by clustering $(\sim+13 \%)$ \\
\hline 500 & Marsden et al. (2009) & $2.3 \pm 0.2$ & BLAST & biased by clustering $(\sim+24 \%)$ \\
\hline \multicolumn{5}{|c|}{ Extrapolated total contribution of the galaxies to the CIB } \\
\hline 24 & Béthermin et al. (2010) & $2.86_{-0.16}^{+0.19}$ & Spitzer/MIPS & direct extraction + stacking \\
\hline 70 & Béthermin et al. (2010) & $6.6_{-0.6}^{+0.7}$ & Spitzer/MIPS & direct extraction + stacking \\
\hline 160 & Béthermin et al. (2010) & $14.6_{-2.9}^{+7.1}$ & Spitzer/MIPS & direct extraction + stacking \\
\hline 850 & Zemcov et al. (2010) & $0.34-0.85$ & SCUBA & lensing survey \\
\hline \multicolumn{5}{|c|}{ Upper limits from $\gamma$-rays absorption } \\
\hline $5-15$ & Renault et al. (2001) & 4.7 & CAT \& HEGRA & $0.5-20 \mathrm{TeV}$ \\
\hline $1-90$ & Mazin\&Raue (2007) & $5-40$ & $\mathrm{TeV}$ exp. & $0.1-20 \mathrm{TeV}$ \\
\hline
\end{tabular}

Table 1: Summary of the measurements of the cosmic infrared background. 


\section{More stringent lower limits from the statistical analysis of far-infrared maps}

In the 200-800 $\mu \mathrm{m}$ range, the faint sources which contribute to the CIB cannot be resolved due to the limited angular resolution of current instruments. Nevertheless, most of these sources are detected in the deepest Spitzer $24 \mu \mathrm{m}$ surveys. The collective signal of these sources, not detected individually in the FIR and sub-mm, can be detected collectively by stacking, providing very stringent lower limits on the CIB in Spitzer/MIPS [4, 23], BLAST [27], PACS [24] bands. It is also possible to compute deep number counts from stacking analysis and to derive an estimation of the total value of the CIB extrapolating the faint end-slope of the number counts [23].

Ultra-deep counts can also be derived from the analysis of the pixel histograms of the infrared maps - $P(D)$ analysis [36, 37]. If the clustering of the galaxies is neglected and the sources are point-like, the pixel histogram depends only on the counts and instrumental properties (instrumental noise and PSF). A non-physical broken power-law description of the number counts can thus be fitted to this pixel histogram $[38,39]$. This method provide deep counts which explains about $2 / 3$ of the CIB at 250,350 and $500 \mu \mathrm{m}$, and is complementary to the stacking analysis. These measurements are summarized in the Fig. 1 and Table 1.

\section{Upper limits from the gamma-ray spectra}

Two photons can interact if the energy in the barycentric frame is sufficient to produce a positron-electron pair. The high-energy $\gamma$ photons can thus be absorbed by the photons of the EBL $[40,41]$. This absorption can be detected as a break at high energy in the spectrum of high-redshift blazars. This phenomenon provides upper limits on the COB and the CIB [29]. Using the spectrum of several blazars, it is now possible to derive stringent upper limits to the CIB below $100 \mu \mathrm{m}$ [30]. The method is somehow dependant on the assumed blazar spectra and on the evolution of the CIB with the redshift [42], but is extremely powerful in particular at near-infrared wavelengths (not covered here).

\section{Conclusion}

The direct measurements of the CIB are difficult because of the accuracy requirements on the foreground subtraction, despite a better knowledge and refined techniques. The lower limits coming from the deep surveys and the upper limits coming from the blazars spectra are more and more stringent, and the association of these two measurements challenges the absolute measurements, especially in the mid-IR, where the zodiacal emission is very strong. Other constraints, e.g. involving the build-up of the CIB with redshift [8] will allow even better observational constraints on the CIB SED and its history, not mentioning the detailed study of the CIB fluctuations (e.g. with Planck at large angular scales [43] and Herschel at small scales [44], following earlier works e.g. with IRAS, ISO, Spitzer, BLAST, Akari). The progress has been amazing since the CIB first detection 15 years ago, the SED being much better known (despite large uncertainties at some wavelengths, like between 30 and 120 microns), and most of the emission being explained 
by directly or indirectly detected galaxy populations, mainly luminous and ultraluminous infrared galaxies.

In the future, these observational constraints will be significantly improved thanks to the next generation of telescopes. In the mid-IR and far-IR, JWST (6.5 m space telescope) will be able to resolve directly almost all the CIB at those wavelengths. These data will also be useful to perform deeper stacking analysis in the sub-mm. Finally, CCAT (25 m sub-mm ground-telescope) and ALMA (sub-mm interferometer) will be able to resolve directly the bulk of the CIB in the sub-mm domain [12], while awaiting possible future space missions dedicated to the study of CIB fluctuations and/or galaxies (e.g. space interferometers in the FIR).

\section{References}

[1] J. Puget, A. Abergel, J. Bernard, F. Boulanger, W. B. Burton, F. Desert, and D. Hartmann, Tentative detection of a cosmic far-infrared background with COBE., A\&A 308 (Apr., 1996) L5+.

[2] D. J. Fixsen, E. Dwek, J. C. Mather, C. L. Bennett, and R. A. Shafer, The Spectrum of the Extragalactic Far-Infrared Background from the COBE FIRAS Observations, ApJ 508 (Nov., 1998) 123-128, [astro-ph/].

[3] M. G. Hauser, R. G. Arendt, T. Kelsall, E. Dwek, N. Odegard, J. L. Weiland, H. T. Freudenreich, W. T. Reach, R. F. Silverberg, S. H. Moseley, Y. C. Pei, P. Lubin, J. C. Mather, R. A. Shafer, G. F. Smoot, R. Weiss, D. T. Wilkinson, and E. L. Wright, The COBE Diffuse Infrared Background Experiment Search for the Cosmic Infrared Background. I. Limits and Detections, ApJ 508 (Nov., 1998) 25-43, [astro-ph/].

[4] H. Dole, G. Lagache, J. Puget, K. I. Caputi, N. Fernández-Conde, E. Le Floc'h, C. Papovich, P. G. Pérez-González, G. H. Rieke, and M. Blaylock, The cosmic infrared background resolved by Spitzer. Contributions of mid-infrared galaxies to the far-infrared background, A\&A 451 (May, 2006) 417-429, [astro-ph/].

[5] M. G. Hauser and E. Dwek, The Cosmic Infrared Background: Measurements and Implications, ARAA 39 (2001) 249-307, [astro-ph/].

[6] G. Lagache, J. Puget, and H. Dole, Dusty Infrared Galaxies: Sources of the Cosmic Infrared Background, ARAA 43 (Sept., 2005) 727-768, [ast ro-ph/].

[7] R. C. Kennicutt, Jr., The Global Schmidt Law in Star-forming Galaxies, ApJ 498 (May, 1998) 541-+, [astro-ph/].

[8] M. Jauzac, H. Dole, E. Le Floc'h, H. Aussel, K. Caputi, O. Ilbert, M. Salvato, N. Bavouzet, A. Beelen, M. Béthermin, J. Kneib, G. Lagache, and J. Puget, The cosmic far-infrared background buildup since redshift 2 at 70 and 160 microns in the COSMOS and GOODS fields, A\&A $\mathbf{5 2 5}$ (Jan., 2011) A52+, [arXiv:1009.0419].

[9] D. Le Borgne, D. Elbaz, P. Ocvirk, and C. Pichon, Cosmic star-formation history from a non-parametric inversion of infrared galaxy counts, A\&A $\mathbf{5 0 4}$ (Sept., 2009) 727-740, [arXiv:0901.3783].

[10] E. Valiante, D. Lutz, E. Sturm, R. Genzel, and E. L. Chapin, A Backward Evolution Model for Infrared Surveys: The Role of AGN- and Color-L $L_{T I R}$ Distributions, ApJ 701 (Aug., 2009) 1814-1838, [arXiv:0906.4110]. 
[11] A. Franceschini, G. Rodighiero, M. Vaccari, S. Berta, L. Marchetti, and G. Mainetti, Galaxy evolution from deep multi-wavelength infrared surveys: a prelude to Herschel, A\&A 517 (July, 2010) A74+.

[12] M. Béthermin, H. Dole, G. Lagache, D. Le Borgne, and A. Pénin, Modeling the evolution of infrared galaxies: A Parametric backwards evolution model, ArXiv e-prints (Oct., 2010) [arXiv:1010.1150].

[13] G. Marsden, E. L. Chapin, M. Halpern, G. Patanchon, D. Scott, M. D. P. Truch, E. Valiante, M. P. Viero, and D. V. Wiebe, A Monte Carlo Approach to Evolution of the Far-Infrared Luminosity Function with BLAST, ArXiv e-prints (Oct., 2010) [arXiv: 1010 .1176].

[14] T. Kelsall, J. L. Weiland, B. A. Franz, W. T. Reach, R. G. Arendt, E. Dwek, H. T. Freudenreich, M. G. Hauser, S. H. Moseley, N. P. Odegard, R. F. Silverberg, and E. L. Wright, The COBE Diffuse Infrared Background Experiment Search for the Cosmic Infrared Background. II. Model of the Interplanetary Dust Cloud, ApJ 508 (Nov., 1998) 44-73, [astro-ph/].

[15] G. Lagache, L. M. Haffner, R. J. Reynolds, and S. L. Tufte, Evidence for dust emission in the Warm Ionised Medium sing WHAM data, A\&A 354 (Feb., 2000) 247-252, [a stro-ph/].

[16] A. Penin and al., An accurate cirrus-free measurement of the intensity and anisotropies of the Cosmic Infrared Background at 100 and 160 microns, submitted to $A \& A$ (2010).

[17] D. J. Fixsen, The Temperature of the Cosmic Microwave Background, ApJ 707 (Dec., 2009) 916-920, [arXiv:0911.1955].

[18] M. Juvela, K. Mattila, D. Lemke, U. Klaas, C. Leinert, and C. Kiss, Determination of the cosmic far-infrared background level with the ISOPHOT instrument, A\&A 500 (June, 2009) 763-768, [arXiv:0904.2997].

[19] S. Matsuura, M. Shirahata, M. Kawada, T. T. Takeuchi, D. Burgarella, D. L. Clements, W. Jeong, H. Hanami, S. A. Khan, H. Matsuhara, T. Nakagawa, S. Oyabu, C. P. Pearson, A. Pollo, S. Serjeant, T. Takagi, and G. White, Detection of the Cosmic Far-Infrared Background in the AKARI Deep Field South, ArXiv e-prints (Feb., 2010) [arXiv: 1002 .3674].

[20] M. Miville-Deschênes, G. Lagache, and J. Puget, Power spectrum of the cosmic infrared background at 60 and $100 \backslash$ umwith IRAS, A\&A 393 (Oct., 2002) 749-756, [astro-ph/].

[21] G. G. Fazio, M. L. N. Ashby, P. Barmby, J. L. Hora, J. Huang, M. A. Pahre, Z. Wang, S. P. Willner, R. G. Arendt, S. H. Moseley, M. Brodwin, P. Eisenhardt, D. Stern, E. V. Tollestrup, and E. L. Wright, Number Counts at $3 \mu \mathrm{m}<\lambda<10 \mu \mathrm{m}$ from the Spitzer Space Telescope, ApJS 154 (Sept., 2004) 39-43, [astro-ph/].

[22] H. I. Teplitz, R. Chary, D. Elbaz, M. Dickinson, C. Bridge, J. Colbert, E. Le Floc'h, D. T. Frayer, J. H. Howell, D. C. Koo, C. Papovich, A. Phillips, C. Scarlata, B. Siana, H. Spinrad, and D. Stern, Spitzer IRS 16 micron Observations of the GOODS Fields, ArXiv e-prints (Oct., 2010) [arXiv: 1010.1797$]$.

[23] M. Béthermin, H. Dole, A. Beelen, and H. Aussel, Spitzer deep and wide legacy mid- and far-infrared number counts and lower limits of cosmic infrared background, A\&A 512 (Mar., 2010) A78+, [arXiv:1001.0896].

[24] S. Berta, B. Magnelli, D. Lutz, B. Altieri, H. Aussel, P. Andreani, O. Bauer, A. Bongiovanni, A. Cava, J. Cepa, A. Cimatti, E. Daddi, H. Dominguez, D. Elbaz, H. Feuchtgruber, N. M. Förster Schreiber, R. Genzel, C. Gruppioni, R. Katterloher, G. Magdis, R. Maiolino, R. Nordon, A. M. Pérez García, A. Poglitsch, P. Popesso, F. Pozzi, L. Riguccini, G. Rodighiero, A. Saintonge, P. Santini, M. Sanchez-Portal, L. Shao, E. Sturm, L. J. Tacconi, I. Valtchanov, M. Wetzstein, and E. Wieprecht, 
Dissecting the cosmic infra-red background with Herschel/PEP, A\&A 518 (July, 2010) L30+, [arXiv:1005.1073].

[25] S. J. Oliver, L. Wang, A. J. Smith, B. Altieri, A. Amblard, V. Arumugam, R. Auld, H. Aussel, T. Babbedge, A. Blain, J. Bock, A. Boselli, V. Buat, D. Burgarella, N. Castro-Rodríguez, A. Cava, P. Chanial, D. L. Clements, A. Conley, L. Conversi, A. Cooray, C. D. Dowell, E. Dwek, S. Eales, D. Elbaz, M. Fox, A. Franceschini, W. Gear, J. Glenn, M. Griffin, M. Halpern, E. Hatziminaoglou, E. Ibar, K. Isaak, R. J. Ivison, G. Lagache, L. Levenson, N. Lu, S. Madden, B. Maffei, G. Mainetti, L. Marchetti, K. Mitchell-Wynne, A. M. J. Mortier, H. T. Nguyen, B. O’Halloran, A. Omont, M. J. Page, P. Panuzzo, A. Papageorgiou, C. P. Pearson, I. Pérez-Fournon, M. Pohlen, J. I. Rawlings, G. Raymond, D. Rigopoulou, D. Rizzo, I. G. Roseboom, M. Rowan-Robinson, M. Sánchez Portal, R. Savage, B. Schulz, D. Scott, N. Seymour, D. L. Shupe, J. A. Stevens, M. Symeonidis, M. Trichas, K. E. Tugwell, M. Vaccari, E. Valiante, I. Valtchanov, J. D. Vieira, L. Vigroux, R. Ward, G. Wright, C. K. Xu, and M. Zemcov, HerMES: SPIRE galaxy number counts at 250, 350, and $500 \mu \mathrm{m}$, A\&A 518 (July, 2010) L21+, [arXiv: 1005 . 2184].

[26] K. Coppin, M. Halpern, D. Scott, C. Borys, J. Dunlop, L. Dunne, R. Ivison, J. Wagg, I. Aretxaga, E. Battistelli, A. Benson, A. Blain, S. Chapman, D. Clements, S. Dye, D. Farrah, D. Hughes, T. Jenness, E. van Kampen, C. Lacey, A. Mortier, A. Pope, R. Priddey, S. Serjeant, I. Smail, J. Stevens, and M. Vaccari, The SCUBA HAlf Degree Extragalactic Survey - VI. 350- $\mu$ m mapping of submillimetre galaxies, MNRAS 384 (Mar., 2008) 1597-1610, [arXiv: 0711 . 0274].

[27] G. Marsden, P. A. R. Ade, J. J. Bock, E. L. Chapin, M. J. Devlin, S. R. Dicker, M. Griffin, J. O. Gundersen, M. Halpern, P. C. Hargrave, D. H. Hughes, J. Klein, P. Mauskopf, B. Magnelli, L. Moncelsi, C. B. Netterfield, H. Ngo, L. Olmi, E. Pascale, G. Patanchon, M. Rex, D. Scott, C. Semisch, N. Thomas, M. D. P. Truch, C. Tucker, G. S. Tucker, M. P. Viero, and D. V. Wiebe, BLAST: Resolving the Cosmic Submillimeter Background, ApJ 707 (Dec., 2009) 1729-1739, [arXiv:0904.1205].

[28] M. Zemcov, A. Blain, M. Halpern, and L. Levenson, Contribution of Lensed SCUBA Galaxies to the Cosmic Infrared Background, ApJ 721 (Sept., 2010) 424-430, [arXiv: 1006.1360 ].

[29] C. Renault, A. Barrau, G. Lagache, and J. Puget, New constraints on the cosmic mid-infrared background using TeV gamma-ray astronomy, A\&A 371 (May, 2001) 771-778, [astro-ph/].

[30] D. Mazin and M. Raue, New limits on the density of the extragalactic background light in the optical to the far infrared from the spectra of all known TeV blazars, A\&A 471 (Aug., 2007) 439-452, [astro-ph/].

[31] D. Elbaz, C. J. Cesarsky, D. Fadda, H. Aussel, F. X. Désert, A. Franceschini, H. Flores, M. Harwit, J. L. Puget, J. L. Starck, D. L. Clements, L. Danese, D. C. Koo, and R. Mandolesi, Source counts from the 15 mи m ISOCAM Deep Surveys, A\&A 351 (Nov., 1999) L37-L40, [astro-ph/].

[32] C. P. Pearson, S. Oyabu, T. Wada, H. Matsuhara, H. M. Lee, S. J. Kim, T. Takagi, T. Goto, M. S. Im, S. Serjeant, M. G. Lee, J. W. Ko, G. J. White, and O. Ohyama, Source counts at 15 microns from the AKARI NEP survey, A\&A 514 (May, 2010) A8+, [arXiv: 1003.2904 ].

[33] H. Dole, G. Lagache, and J. Puget, Predictions for Cosmological Infrared Surveys from Space with the Multiband Imaging Photometer for SIRTF, ApJ 585 (Mar., 2003) 617-629, [ast ro-ph/].

[34] H. Dole, G. H. Rieke, G. Lagache, J. Puget, A. Alonso-Herrero, L. Bai, M. Blaylock, E. Egami, C. W. Engelbracht, K. D. Gordon, D. C. Hines, D. M. Kelly, E. Le Floc'h, K. A. Misselt, J. E. Morrison, J. Muzerolle, C. Papovich, P. G. Pérez-González, M. J. Rieke, J. R. Rigby, G. Neugebauer, J. A. Stansberry, K. Y. L. Su, E. T. Young, C. A. Beichman, and P. L. Richards, Confusion of Extragalactic Sources in the Mid-and Far-Infrared: Spitzer and Beyond, ApJS 154 (Sept., 2004) 93-96. 
[35] B. Altieri, S. Berta, D. Lutz, J. Kneib, L. Metcalfe, P. Andreani, H. Aussel, A. Bongiovanni, A. Cava, J. Cepa, L. Ciesla, A. Cimatti, E. Daddi, H. Dominguez, D. Elbaz, N. M. Förster Schreiber, R. Genzel, C. Gruppioni, B. Magnelli, G. Magdis, R. Maiolino, R. Nordon, A. M. Pérez García, A. Poglitsch, P. Popesso, F. Pozzi, J. Richard, L. Riguccini, G. Rodighiero, A. Saintonge, P. Santini, M. Sanchez-Portal, L. Shao, E. Sturm, L. J. Tacconi, I. Valtchanov, M. Wetzstein, and E. Wieprecht, Herschel deep far-infrared counts through Abell 2218 cluster-lens, A\&A 518 (July, 2010) L17+, [arXiv:1005.1575].

[36] J. J. Condon, Confusion and Flux-Density Error Distributions, ApJ 188 (Mar., 1974) 279-286.

[37] Y. Friedmann and F. Bouchet, Fluctuation analysis of the far-infrared background - information from the confusion, MNRAS 348 (Mar., 2004) 737-744, [astro-ph/].

[38] G. Patanchon, P. A. R. Ade, J. J. Bock, E. L. Chapin, M. J. Devlin, S. R. Dicker, M. Griffin, J. O. Gundersen, M. Halpern, P. C. Hargrave, D. H. Hughes, J. Klein, G. Marsden, P. Mauskopf, L. Moncelsi, C. B. Netterfield, L. Olmi, E. Pascale, M. Rex, D. Scott, C. Semisch, N. Thomas, M. D. P. Truch, C. Tucker, G. S. Tucker, M. P. Viero, and D. V. Wiebe, Submillimeter Number Counts from Statistical Analysis of BLAST Maps, ApJ 707 (Dec., 2009) 1750-1765, [arXiv: 0906.0981$].$

[39] J. Glenn, A. Conley, M. Béthermin, B. Altieri, A. Amblard, V. Arumugam, H. Aussel, T. Babbedge, A. Blain, J. Bock, A. Boselli, V. Buat, N. Castro-Rodríguez, A. Cava, P. Chanial, D. L. Clements, L. Conversi, A. Cooray, C. D. Dowell, E. Dwek, S. Eales, D. Elbaz, T. P. Ellsworth-Bowers, M. Fox, A. Franceschini, W. Gear, M. Griffin, M. Halpern, E. Hatziminaoglou, E. Ibar, K. Isaak, R. J. Ivison, G. Lagache, G. Laurent, L. Levenson, N. Lu, S. Madden, B. Maffei, G. Mainetti, L. Marchetti, G. Marsden, H. T. Nguyen, B. O’Halloran, S. J. Oliver, A. Omont, M. J. Page, P. Panuzzo, A. Papageorgiou, C. P. Pearson, I. Pérez-Fournon, M. Pohlen, D. Rigopoulou, D. Rizzo, I. G. Roseboom, M. Rowan-Robinson, M. S. Portal, B. Schulz, D. Scott, N. Seymour, D. L. Shupe, A. J. Smith, J. A. Stevens, M. Symeonidis, M. Trichas, K. E. Tugwell, M. Vaccari, I. Valtchanov, J. D. Vieira, L. Vigroux, L. Wang, R. Ward, G. Wright, C. K. Xu, and M. Zemcov, HerMES: deep galaxy number counts from a $P(D)$ fluctuation analysis of SPIRE Science Demonstration Phase observations, MNRAS 409 (Nov., 2010) 109-121, [arXiv: 1009.5675$].$

[40] G. G. Fazio and F. W. Stecker, Predicted High Energy Break in the Isotropic Gamma Ray Spectrum: a Test of Cosmological Origin, Nature 226 (Apr., 1970) 135-136.

[41] M. Raue, T. Kneiske, and D. Mazin, First stars and the extragalactic background light: how recent $\gamma$-ray observations constrain the early universe, A\&A 498 (Apr., 2009) 25-35, [arXiv:0806.2574].

[42] T. M. Kneiske and H. Dole, A lower-limit flux for the extragalactic background light, A\&A 515 (June, 2010) A19+, [arXiv:1001.2132].

[43] Planck Collaboration, et al. 2011, arXiv:1101.2028

[44] Amblard, A., et al. 2011, arXiv:1101.1080 